\title{
Distribuição espacial dos idosos no município de Botucatu segundo o grau de atividade física em atividades de recreação e lazer
}

Spatial distribution of aged people in the city of Botucatu according to their level of physical activity in recreation and leisure activities

Distribución espacial de los ancianos en la ciudad de Botucatu en el grado de actividad física de ocio y tiempo libre

Fabia Tetsue Bicalho Yamaguti', Tania Ruiz², Ligia Vizeu Barrozo ${ }^{3}$, José Eduardo Corrente ${ }^{4}$

Palavras-chave:

Atividade Motora

Distribuição Espacial da População

\section{Resumo}

Introdução: À medida que a população envelhece, cresce a preocupação com a manutenção de sua qualidade de vida e capacidade funcional, as quais estão associadas ao grau de atividade física mantida. 0 uso de técnicas de geoprocessamento possibilita a análise espacial de dados de saúde, relacionando-os às características da população estudada, 0 que permite 0 direcionamento de ações a grupos de risco específicos. Objetivo: Estudar a distribuição espacial da população de 60 anos e mais do município de Botucatu, segundo 0 grau de atividade física. Métodos: Foi realizada uma amostragem sistemática populacional por famílias. Dessas que possuíam idosos, foram sorteados 365 correspondentes a uma prevalência de $50 \%$ com uma margem de erro de $5 \%$ e confiança de 95\%. A esses idosos, foram aplicados vários instrumentos para avaliar a qualidade e o estilo de vida, dentre eles o International Physical Activities Questionaire (IPAQ). Utilizando o domínio de atividades físicas, esporte, recreação e lazer, esses idosos foram classificados como muito ativos, ativos, irregularmente ativos e sedentários, como proposto por Matsudo (2001). Para avaliar a distribuição espacial dos idosos, segundo o grau de atividade física de lazer, utilizou-se o índice de Moran. As análises foram feitas por meio do programa GeoDa. Resultados: Observou-se que o grau de atividade física é baixo e que existe associação entre os locais de oferta para a prática de esportes, topografia e o grau de atividade física. Não foi observada relação entre o grau de atividade física e o sexo ou a idade dos entrevistados. Conclusão: Aumento e melhor distribuição da estrutura para a prática de atividade física ajudariam a melhorar a atividade desta faixa etária e, portanto, a qualidade de vida.

Keywords:

Aged

Motor Activity

Residence Characteristics

\section{Abstract}

Introduction: As the population ages, there is increasing concern about the maintenance of people's quality of life and functional capacity, which are associated with their level of activity performed. The use of geoprocessing techniques enables the spatial analysis of health data by relating them to the characteristics of the studied population, and this allows targeting actions at specific risk groups. Objective: To study the spatial distribution of the population of individuals aged 60 years-old and over in the city of Botucatu, in São
Faculdade de Medicina de Botucatu (UNESP). bubsxli@yahoo.com.br

${ }^{2}$ Faculdade de Medicina de Botucatu (UNESP). truiz@fmb.unesp.br

${ }^{3}$ Faculdade de Filosofia, Letras e Ciências Humanas (USP). lija@usp.br ${ }^{4}$ Departamento de Bioestatística - IB (UNESP). jecorren@ibb.unesp.br

${ }^{\star}$ Autor correspondente.

Fonte de financiamento: FAPESP Processo no 04/06881-0.

Conflito de interesses: declararam não haver.

Recebido em: 10/06/2011

Aprovado em: 16/10/2011 
Paulo, according to their level of physical activity. Methods: A systematic population sampling by families was performed. Of the families comprising elderly members, 365 were randomly selected, corresponding to a prevalence of $50 \%$ with a margin of error of $5 \%$ and confidence of $95 \%$. Several instruments were applied to the older individuals in order to evaluate quality of life and lifestyle, such as the International Physical Activity Questionnaire (IPAQ). By using the domain of physical activities, sports, recreation and leisure, the older individuals were classified as very active, active, irregularly active, and sedentary, according to Matsudo (2001). To evaluate the older people's spatial distribution based on their level of leisure physical activity, Moran's index was used. Analyses were performed by the GeoDa software. Results: It was observed that the level of physical activity was low, and that there was an association between the sites provided for sports practice, topography, and the level of physical activity. No relation was observed between physical activity level and the respondents' gender or age. Conclusion: Increase and better distribution of the structure for physical activity practice would help improving physical activity at this age range and, therefore, the individuals' quality of life.

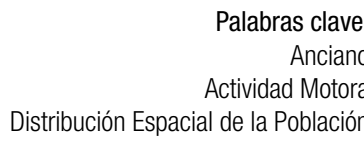

\section{Resumen}

Introducción: A medida que la población envejece, una creciente preocupación por el mantenimiento de su calidad de vida y capacidad funcional, que se asocian con el grado de actividad física mantenida. El uso de técnicas de SIG permite el análisis espacial de datos de salud, en relación a las características de la población estudiada, lo que permite las acciones directas de los grupos de riesgo específicos. Objetivo: Estudiar la distribución espacial de la oblación de 60 años o más de Botucatu, de acuerdo a su grado de actividad física. Métodos: Se realizó un muestreo sistemático por hogar. De los que tenían más edad, se extrajeron 365 que corresponde a una prevalencia del $50 \%$ con un margen de error del $5 \%$ y el $95 \%$ de confianza. A estos ancianos, se les aplicó diversos instrumentos para evaluar la calidad y el estilo de vida, incluyendo el Cuestionario Internacional de Actividad Física (IPAQ). Mediante el dominio de la actividad física, deporte, recreación y esparcimiento, estos ancianos fueron clasificados como muy activos, activos, de forma irregular activa y sedentarios, según lo que fue propuesto por Matsudo (2001). Para evaluar la distribución espacial de las personas mayores en función del grado de actividad física de ocio, se utilizó el índice de Moran. El análisis se hizo a través de la GeoDa programa. Resultados: Se observó que el grado de actividad física es bajo y que existe una asociación entre la oferta local de los deportes, la topografía y el grado de actividad física. No se encontró relación entre el grado de actividad física y el sexo o la edad de los encuestados. Conclusión: El aumento y una mejor distribución del marco de la actividad física ayudarían a mejorar la actividad de este grupo de edad y por lo tanto su calidad de vida.

\section{Introdução}

O crescente envelhecimento da população de todo o mundo tem sido um dos maiores desafios da área de saúde pública neste século. A literatura que trata dos problemas relacionados com a população idosa no Brasil enfatiza que está sendo experimentando um aumento significativo na proporção de idosos, o qual chega a mais de $10 \%$ da população em alguns estados ${ }^{1}$. A preocupação tem enfocado o indivíduo idoso, que tem menor capacidade funcional e autonomia, uma vez que considera-se a manutenção da independência para a realização das atividades diárias como fator preditivo do envelhecimento bem-sucedido ${ }^{2}$.

Embora doenças incapacitantes sejam sabidamente responsáveis pela diminuição da capacidade funcional, estudos têm demonstrado uma associação de fatores socioeconômicos, culturais, demográficos e psicossociais nesse processo, dentre eles o sedentarismo ${ }^{3}$.

O sedentarismo ou o nível insuficiente de atividade física são condições que prevalecem na população geral ${ }^{4}$, estando associados ao maior risco de doenças crônico-degenerativas ${ }^{5}$ e à acentuação da perda de força muscular natural do envelhecimento, determinando, assim, um declínio da aptidão física e, consequentemente, da capacidade funcional ${ }^{6}$.
Além de ter papel protetor sobre a saúde ${ }^{7}$, a prática de exercícios físicos regulares proporciona ao idoso a oportunidade de fazer novas amizades, manter o convívio social e a interação com outras pessoas, além de aumentar a flexibilidade, o equilíbrio e o tônus muscular, o que auxilia na prevenção de quedas - grandes causadoras da incapacidade entre os mais velhos ${ }^{8}$.

Há evidências, ainda, de que a prática de atividades físicas também contribua para a melhora da autoestima e da cognição, reduzindo o risco de doenças demenciais, bem como de ansiedade e de sintomas depressivos?

Em virtude desses fatores é que diversas políticas públicas têm sido formuladas, com o intuito de permitir que a população permaneça ativa e independente à medida que envelhece ${ }^{10}$. Tais políticas se baseiam em dados primários de saúde, colhidos de forma coletiva e que representam os fenômenos presentes na população, mas não possibilitam a individualização do seu gerenciamento, pois esta não apresenta homogeneidade em sua distribuição ${ }^{11}$.

Ainda são poucos os estudos epidemiológicos que utilizam a técnica de geoprocessamento e que indicam o perfil da população idosa no Brasil. Esta técnica pode ser definida como um conjunto de tecnologias de coleta, tratamento, manipulação e apresentação de informações espaciais, as quais possibilitam localizar os indivíduos no espaço e associar suas características ao local em que vivem ${ }^{12}$. 
O uso da técnica de geoprocessamento, além de proporcionar maior facilidade de manutenção do banco de dados, possibilita a rápida atualização de mapas, sem a necessidade de refazê-los manualmente. Permite ainda a identificação dos padrões espaciais de morbidade e mortalidade e os fatores associados a esses padrões ${ }^{13}$, podendo ser aplicado na associação imediata entre quaisquer indicadores presentes em um banco de dados.

O desenvolvimento de análises baseadas na distribuição espacial dos eventos pode trazer uma contribuição interessante para a gestão de um sistema local de saúde ao direcionar suas atividades ${ }^{14}$. Esta contribuição será ainda mais significativa em áreas densamente povoadas, nas quais verifica-se uma complexa disposição dos diversos segmentos sociais, os quais dificultam a identificação de vulnerabilidades localizadas ${ }^{15}$.

O objetivo do presente estudo foi obter a distribuição espacial dos idosos no município de Botucatu, segundo seu grau de atividade física.

\section{Material e métodos}

Um inquérito no município de Botucatu, em 2003, envolveu 365 indivíduos com mais de 60 anos, residentes na zona urbana ${ }^{15}$. Por meio de um instrumento multidimensional, foram avaliados, em vários módulos, os aspectos referentes à qualidade de vida, tais como: níveis de atividade física e perguntas sobre seu grau de satisfação com a vida.

A amostra, de base populacional, foi calculada para prevalência desconhecida de $50 \%$. A amostragem foi sistemática e, a cada quatro residências, selecionou-se a última das quatro. Foram, então, identificadas, nesta amostra, as residências que possuíam indivíduos com 60 anos ou mais. Esses idosos foram listados e, por meio da tabela de números aleatórios, selecionaram-se 365, conforme cálculo do tamanho da amostra. Iniciou-se, desta forma, o banco de dados, completado após as entrevistas domiciliares.

Os dados desse cadastro, com formato Excel, foram então transportados para o programa SPSS, que possibilitou a identificação dos idosos de acordo com a classificação original proposta por Matsudo et al. ${ }^{16}$, os quais dividem os idosos nas seguintes categorias:

1. Muito ativo: aquele que cumpriu as recomendações de:

- atividade vigorosa - $\geq 5$ dias/ semana e $\geq 30$ minutos por sessão e/ou;

- atividade vigorosa - $\geq 3$ dias/ semana e $\geq 20$ minutos por sessão + atividade moderada e/ou caminhada $\geq 5$ dias/ semana e $\geq 30$ minutos por sessão.
2. Ativo: aquele que cumpriu as recomendações de:

- atividade vigorosa $-\geq 3$ dias / semana e $\geq 20$ minutos por sessão e/ou;

- atividade vigorosa $-\geq 5$ dias/ semana e $\geq 30$ minutos por sessão e/ou;

- qualquer atividade somada $-\geq 5$ dias/ semana e $\geq 150$ minutos/ semana (caminhada + atividade moderada + atividade vigorosa).

3. Irregularmente ativo: aquele que realiza atividade física, porém insuficiente para ser classificado como ativo, pois não cumpre as recomendações quanto à frequência ou à duração. Para realizar essa classificação, soma-se a frequência e a duração dos diferentes tipos de atividades (caminhada + atividade moderada + atividade vigorosa).

- Frequência - cinco dias por semana;

- Duração - 150 minutos por semana;

- Aquele que não atingiu nenhum dos critérios da recomendação quanto à frequência nem quanto à duração.

4. Sedentário: aquele que não realizou nenhuma atividade física por pelo menos dez minutos contínuos durante a semana.

Em uma primeira análise, constatou-se que, levando-se em consideração as atividades relatadas pelos idosos, a maioria enquadrava-se como ativo ou muito ativo. Entretanto, averiguou-se que o International Physical Activities Questionnaire (IPAQ) não foi validado especificamente para esta faixa etária, pois os domínios de atividade física no trabalho, como meio de transporte, tarefas domésticas e cuidado da família, não contribuíam para o índice por características da faixa etária estudada. Optou-se, deste modo, avaliar somente as atividades físicas de recreação, esporte, exercício e lazer realizadas pelos idosos, usando-se os mesmos critérios para classificação propostos por Matsudo et al. ${ }^{16}$.

Quantificou-se o número de idosos pertencentes à cada categoria por setor censitário e, posteriormente, calculou-se a proporção de idosos de cada classe de atividade (inativo, irregularmente ativo, ativo e muito ativo) em relação ao total de idosos amostrados por setor. Os setores não contemplados com nenhum idoso amostrado foram incluídos no setor vizinho mais próximo ao qual fazia parte, de acordo com o censo de 1996. Assim, o mapa da cidade totalizou 129 setores, cada um com pelo menos um idoso amostrado, evitando-se alguns sem dados.

A análise espacial foi do tipo univariada, ou seja, um único tipo de classe de atividade foi avaliado por vez, utilizando-se as proporções já calculadas por setor censitário. O padrão na distribuição espacial pode ser classificado de três formas: uniforme, em conglomerados ou aleatória. Para medir a probabilidade de um padrão espacial aparente ter sido 
produzido meramente ao acaso, utilizou-se o coeficiente I de Moran ${ }^{17}$, que indica se os dados apresentam dependência espacial (autocorrelação espacial) e mede o padrão global de distribuição espacial, indicando se há uma tendência geral para a distribuição do evento que se estuda.

O coeficiente I é similar ao da correlação de Pearson. Na autocorrelação espacial, a correlação é examinada entre diferentes valores dentro da mesma variável, e uma matriz de ponderação é incluída para definir as relações espaciais entre as áreas. O coeficiente I é calculado como a razão do produto do desvio médio e as somas dos quadrados das coordenadas $x$ (longitude) e $y$ (latitude) (Equação 1):

$$
I=\frac{N \sum_{i} \sum_{j} w_{i j}\left(x_{i}-\bar{x}\right)\left(x_{j}-\bar{x}\right)}{J \sum_{i} \sum_{j} w_{i j} \sum_{i}\left(x_{i}-\bar{x}\right)^{2}}
$$

onde,

N é o número de setores censitários no estudo,

$J$ o número de pares de setores examinados, $x_{\mathrm{i}}$ o valor (prevalência) do setor $i$,

$x_{\mathrm{j}}$ é o valor do setor $j\left(x_{\mathrm{i}}\right.$ e $x_{\mathrm{j}}$ são dois setores de vizinhos próximos) e

$w_{\mathrm{ij}}$ é a matriz de ponderação.

O coeficiente I de Moran varia de -1 a 1 , sendo 0 quando a inexistência de autocorrelação (não há efeito da distância sobre a distribuição da variável) é verificada. Um valor positivo de I implica em agrupamento e um negativo, em dispersão. $\mathrm{O}$ valor esperado de I é -1/(N-1), que se aproxima de 0 à medida que $\mathrm{N}$ aumenta. Para verificar a inexistência de autocorrelação, utilizase a estatística $z$, que pode ser calculada da maneira convencional, usando-se o desvio padrão de I, $\sigma_{\mathrm{I}}$, já que a média se aproxima de zero e a distribuição de $z$ é aproximadamente normal.

O coeficiente I para cada classe de atividade foi calculado por meio do programa $\mathrm{GeoDa}^{18}$.

Embora seja capaz de apontar a tendência geral de agrupamento dos dados, o coeficiente I de Moran é uma medida global e, por isso, não revela padrões locais de associação espacial. Para verificar esta estrutura, foram utilizados indicadores locais de associação espacial, Local Indicators of Spatial Association (LISA) ${ }^{18}$. Estes são usados para testar a hipótese nula de distribuição aleatória por comparar os valores de cada localização específica com os de seus vizinhos. A estatística LISA também foi calculada por meio do programa Geoda, sendo apropriada para identificar agrupamentos espaciais significativos.

A estrutura de vizinhança pode ser estabelecida por meio de matrizes de pesos espaciais. Neste estudo, foram estabelecidas matrizes de pesos de contiguidade binária, segundo convenção (rook).
Os coeficientes de autocorrelação espacial global e associação local foram considerados significativos quando $\mathrm{p}<0,05$. Como os eventos eram referentes a taxas (proporções), foram aplicados os testes de Moran com Estimativa Bayesiana Empírica para taxas. Quando o número de pessoas expostas é pequeno, qualquer leve alteração no número de eventos, mesmo que devido ao mero acaso, provoca grandes variações no valor da taxa. Marshall ${ }^{19}$ propôs a aplicação de Estimadores Bayesianos Empíricos como uma alternativa ao uso de taxas brutas nessas condições. Essa estimativa calcula a média ponderada entre a taxa bruta para cada setor e a de uma janela espacial, com pesos proporcionais à população em risco (número de idosos amostrados por setor), resultando num mapa suavizado.

Com base nos resultados obtidos pelos indicadores LISA para cada classe de atividade, elaborou-se um mapa com os setores onde ocorriam agrupamentos significativos para todas as classes. Para a elaboração desse mapa, utilizou-se o programa IDRISI32.

Os procedimentos foram aprovados pelo Comitê de Ética da Faculdade de Medicina de Botucatu, conforme resolução CNS 196/96 (Processo nº 1090/2002).

\section{Resultados}

Os dados estão descritos com a mesma classificação de Matsudo ${ }^{16}$, considerando somente o domínio de atividade física, lazer e recreação. Observou-se que 1,64\% ( $\mathrm{n}=6)$ dos idosos eram muito ativos, $19,18 \%(\mathrm{n}=70)$ eram ativos, $21,37 \%(\mathrm{n}=78)$ eram irregularmente ativos e $57,81 \%$ $(\mathrm{n}=211)$ eram sedentários.

Não foi observada uma associação significativa entre o grau de atividade física do idoso e o sexo ou faixa etária.

Os coeficientes I de Moran foram calculados, levando-se em conta cada classe de atividade física. Os valores são apresentados na Tabela 1 , na qual observa-se que apenas a classe de inativos apresenta um padrão espacial significativo.

A associação espacial local, medida pela estatística LISA, pode ser observada na Tabela 2.

Embora os valores estejam expressos na Tabela 2, os resultados pouco significam se não forem visualizados em um mapa. A Figura 1 apresenta o mapa dos setores que formam agrupamentos significativos para cada classe de atividade entre os maiores de 60 anos, no município de Botucatu.

A Figura 2 mostra a localização das academias, escolas de natação, clubes e áreas de lazer destinadas à prática de atividades físicas em Botucatu. 
Tabela 1. Indicador global de autocorrelação espacial para cada classe de atividade física entre os idosos de 60 anos e mais (Botucatu, 2003).

\begin{tabular}{lcc}
\hline Classe de atividade & Coeficiente I & Valor $p$ \\
\hline Inativo & 0,0768 & 0,002 \\
\hline Irregularmente ativo & 0,0131 & 0,321 \\
Ativo & 0,0117 & 0,327 \\
\hline Muito ativo & $-0,0045$ & 0,552 \\
\hline
\end{tabular}

Figura 1. Mapa dos setores significativos para cada classe de atividade entre os maiores de 60 anos, no município de Botucatu, de acordo com o inquérito de 2003.

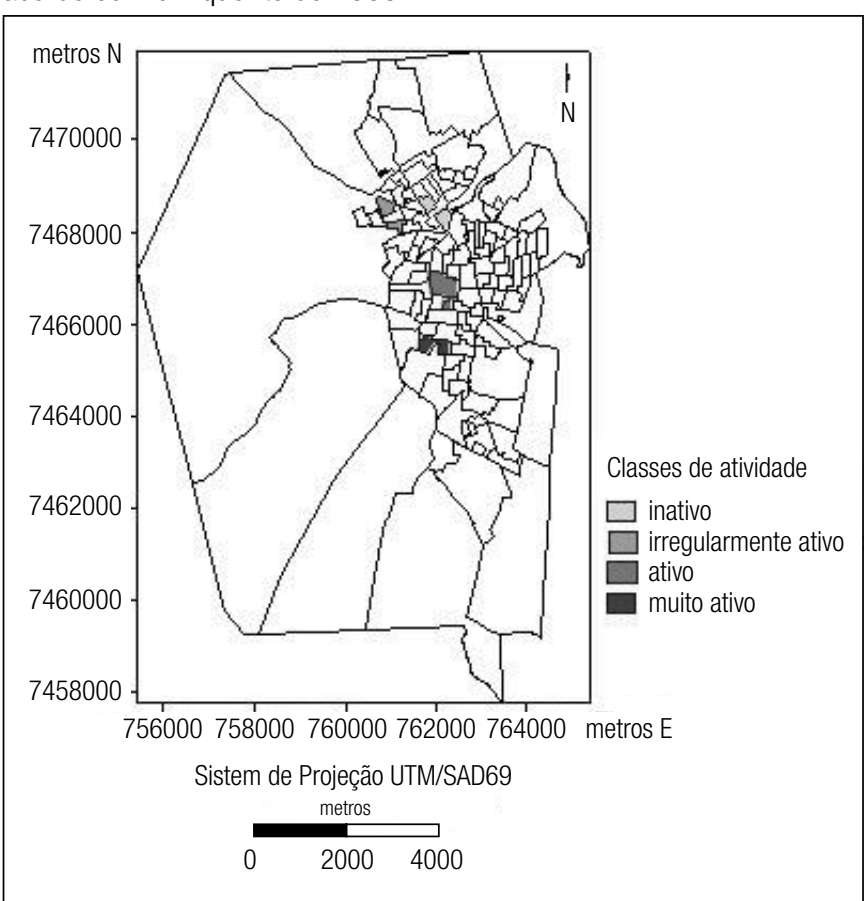

A análise das figuras mostra que setores significativos para a classe de "inativos" correspondem a uma porção da cidade (Vila dos Lavradores), com características desfavoráveis, rampas bastante inclinadas e pouca oferta de locais que disponibilizem a prática de atividades físicas. A classe de "irregularmente ativos" corresponde a bairros (Vila Antártica e Vila Casa Branca) com oferta muito pobre de estabelecimentos destinados ao esporte e lazer e, ainda, de topografia irregular, sendo uma extensão da área citada anteriormente e, portanto, mantendo algumas características semelhantes a ela. Os setores classificados como "ativos" referem-se ao Centro da cidade, que, além de apresentar extensa área plana, conta com maior disponibilidade de meios de transporte e possui bom aporte de locais destinados à prática de atividades físicas. O setor muito ativo (parte do Bairro Lavapés e Vila Santana), por sua vez, apesar de não ter uma topografia tão favorável, apresenta alta concentração de academias, escolas de natação, clubes e áreas de lazer.
Tabela 2. Indicador local de associação espacial para cada classe de atividade entre os maiores de 60 anos de idade.

\begin{tabular}{lcc}
\hline Classe de atividade & Setores & Valor $p$ \\
\hline Inativo & 63 e 90 & $<0,05$ e $<0,05$ \\
Irregularmente ativo & 117 e 86 & $<0,05$ e $<0,05$ \\
Ativo & 5 e 3 & $<0,01$ e $<0,01$ \\
Muito ativo & 77 & $<0,05$ \\
\hline
\end{tabular}

Figura 2. Mapa dos setores significativos para cada classe de atividade entre os maiores de 60 anos e locais para a prática de atividades físicas no município de Botucatu.

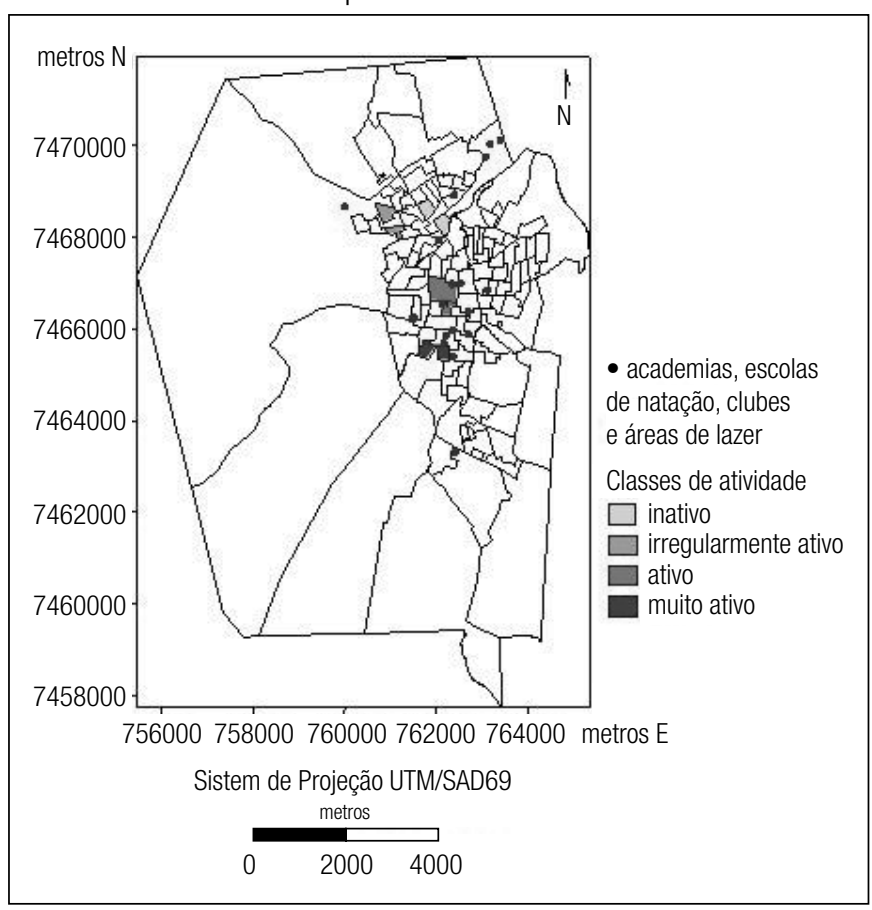

\section{Discussão}

Os dados obtidos sugerem que a oferta de locais para a prática de esportes e a topografia do local podem ser responsáveis pelo incentivo à prática de esportes.

Os registros dos Sistemas de Informação em Saúde (SIS), tendo como escala geográfica o município ou os bairros, têm sido utilizados como informação importante de situações associadas a eventos de morbimortalidade ou de prevenção. Porém, em alguns casos, como nas regiões densamente povoadas, essa escala tem sido insuficiente devido à necessidade de enfocar áreas menores. Diante desse fato, alguns estudos têm trabalhado os dados segundo os setores censitários, por meio do georreferenciamento dos endereços dos indivíduos avaliados ${ }^{20}$.

Tal proposta mostrou-se interessante sob o ponto de vista teórico, já que possibilitaria uma visão mais fidedigna da disposição dos indivíduos no espaço em que vivem. Na prática, 
no entanto, apresentou alguns inconvenientes, como a irregularidade da numeração das residências, que não seguem um padrão lógico (crescente ou decrescente), e a desatualização do mapa fornecido pelos Correios, com a numeração das casas. Esse problema também já foi observado em outros estudos e talvez se torne um dos principais empecilhos na implementação de seu uso numa escala mais ampla, já que diversas cidades também apresentam padrão de numeração irregular ou utilizam endereços por quadra e não por logradouro ${ }^{21}$.

Os resultados obtidos neste estudo exemplificam como a análise geográfica pode ser útil na Saúde Pública, pois permite a compreensão de como um contexto pode afetar a saúde de grupos populacionais que estão submetidos a processos de seleção, interação e adaptação, fornecendo respostas que não podem ser medidas no nível individual, mas por meio de uma abordagem ecológica ${ }^{22}$. O contexto ambiental (no caso, a topografia e a oferta de academias), na medida em que contribui com o surgimento de agravos à saúde (sedentarismo), possibilita a identificação de grupos populacionais que estão submetidos a riscos de natureza difusa, muitas vezes superpostos, expressandose em características socioeconômicas e demográficas ${ }^{22}$. O estrato social dos idosos está associado aos bairros e aglomerados encontrados neste estudo e que foram historicamente determinados no crescimento do município. Mesmo levando-se em conta que a infraestrutura disponível topograficamente está associada ao grau de atividade física dos idosos, entende-se que o estrato social foi fundamental no incentivo à prática de esportes e, neste caso, refletido no bairro onde tais pessoas moram.

O que reforça a constatação de que o estrato social é um dos fatores mais importantes no grau de atividade física desempenhada pelos idosos é o fato de não ter sido identificada uma associação significativa deste evento com o sexo e a idade.

Desse modo, conclui-se que o uso do geoprocessamento contribui para produção de informação, o que é útil na saúde pública e leva à melhoria da qualidade de vida da população.

No caso dos idosos de Botucatu, poderá ser motivado o delineamento de projetos que incluam atividades físicas direcionadas especificamente para esse público, enfocando as áreas onde estes foram classificados como "inativos" ou "irregularmente ativos".

\section{Referências}

1. Dias JR, Costa CS, Lacerda MA. O envelhecimento da população brasileira: uma análise de conteúdo das páginas da REBEP. Rev Bras Geriatr Gerontol. 2006; 9(2): 1-21.
2. Moraes JLD, Souza VBA. Factors associated with the successfull aging of the socially-active elderly in the metropolitan region of Porto Alegre. Rev Bras Psiquiatr. 2005; 27(4): 302-8. http://dx.doi.org/10.1590/S151644462005000400009

3. RosaTEC, Benício MHD, Latorre MRDO, Ramos LR. Fatores determinantes da capacidade funcional entre idosos. Rev Saúde Pública. 2003; 37(1): 40-8. http://dx.doi.org/10.1590/S0034-89102003000100008

4. Brasil; Ministério da Saúde; Secretaria de Vigilância em Saúde Secretaria de Atenção à Saúde; Instituto Nacional de Câncer, Coordenação de Prevenção e Vigilância. Inquérito domiciliar sobre comportamentos de risco e morbidade referida de doenças e agravos não transmissíveis: Brasil, 15 capitais e Distrito Federal, 2002-2003. Rio de Janeiro: INCA; 2004.

5. Organização Pan-Americana da Saúde. Doenças crônico-degenerativas e obesidade: estratégia mundial sobre alimentação saudável, atividade física e saúde. Brasília; 2003.

6. Matsudo SM, Keihan V, Matsudo R, Barros Neto TL. Impacto do envelhecimento nas variáveis antropométricas, neuromotoras e metabólicas da aptidão física. Rev Bras Cienc Mov. 2000; 8(1): 15-21.

7. Associação Médica Brasileira, Conselho Federal de Medicina, Sociedade Brasileira de Medicina do Esporte. Projeto Diretrizes. Atividade física e saúde. São Paulo; 2001.

8. World Health Organization [Internet]. Physical activity and older people. [acesso em 2007 Ago 04]. Disponível em: http://www.who.int/ moveforhealth/advocacy/information_sheets/elderly/en/index.html

9. Matsudo SM. Atividade física na promoção da saúde e qualidade de vida no envelhecimento. Rev Bras Educ Fis Esp. 2006; 20 (Suppl 5): S135-7.

10. World Health Organization. Envelhecimento ativo: uma política de saúde / World Health Organization; tradução Suzana Gontijo. Brasília: Organização Pan-Americana da Saúde; 2005.

11. Bezerra Filho JG, Kerr LRFS, Miná DL, Barreto ML. Distribuição espacial da taxa de mortalidade infantil e principais determinantes no Ceará Brasil, no período 2000-2002. Cad Saúde Pública. 2007; 23(5): 1173-85. http://dx.doi.org/10.1590/S0102-311X2007000500019

12. Pina MFRP. Potencialidades dos Sistemas de Informações Geográficas na Área da Saúde. In: Najar AL \& Marques EC. Saúde e Espaço: Estudos metodológicos e técnicas de análise. Rio de Janeiro: Editora Fiocruz; 1998.

13. Carvalho MS. Aplicação de métodos de análise espacial na caracterização de áreas de risco a saúde [tese de doutorado]. Engenharia Biomédica, COPPE/ UFRJ. Rio de Janeiro; 1997.

14. Hino P, Santos CB, Villa TCS. Evolução espaço-temporal dos casos de tuberculose em Ribeirão Preto (SP), nos anos de 1998 a 2002. J Bras Pneumol. 2005; 31(6): 523-7. http://dx.doi.org/10.1590/S180637132005000600011

15. Jóia LC, Ruiz T, Donalisio MR. Condições associadas ao grau de satisfação com a vida entre a população de idosos. Rev Saúde Pública. 2007; 41(1): 131-8. http://dx.doi.org/10.1590/S0034-89102007000100018

16. Matsudo SM, Araujo T, Matsudo V, Andrade D, Andrade E, Oliveira LC et al. Questionário internacional de atividade física (IPAQ): estudo de validade e reprodutibilidade no Brasil. Rev Bras Ativ Fis Saúde. 2001; 6(2): 5-18.

17. Moran PAP. Notes on continuous stochastic phenomenon. Biometrika 1950; 37: 17-23. http://dx.doi.org/10.2307/2332142

18. Anselin L. GeoDa 0.9 User's Guide. Spatial Analysis Laboratory (SAL). Department of Agricultural and Consumer Economics. Illinois: University of Illinois, Urbana-Champaign, IL; 2003.

19. Marshall RJ. Mapping disease and mortality rates using Empirical Bayes estimators. Appl Statist. 1991; 40(2): 283-94. http://dx.doi. org/10.2307/2347593

20. Skaba DA, Carvalho MS, Barcellos C, Martins PC, Terron SL. Geoprocessamento dos dados da saúde: o tratamento dos endereços. Cad Saúde Pública. 2004; 20(6): 1753-6. http://dx.doi.org/10.1590/ S0102-311X2004000600037

21. Susser M. The Logic in Ecological: I. The Logic of Analysis. Am J Pub Health. 1994; 84(5): 825-9. http://dx.doi.org/10.2105/AJPH.84.5.825

22. Barcellos C, Bastos FI. Geoprocessamento, ambiente e saúde: uma união possível? Cad Saúde Pública. 1996; 12(3): 389-97. http://dx.doi. org/10.1590/S0102-311X1996000300012 